SECTION 26. Radio-technique. Electronics. Telecommunications.

Andrei Sergeevich Salnikov

Junior research associate

Tomsk State University of Control Systems and Radioelectronics, Russia ansalnikov@gmial.com

\title{
BEHAVIORAL MODEL BUILDING FOR MICROWAVE INTEGRATED CIRCUIT PASSIVE ELEMENTS
}

\begin{abstract}
In the article results of scalable model building for microwave monolithic integrated circuits elements are presented. The inverse distant weighting and an artificial neural network were used to build models. Behavioral models of thin-film resistor, MIM capacitor, and spiral square inductor were built. Comparison of the models by accuracy, calculation speed and building complexity is presented. network.

Key words: MMIC elements, behavioral model, inverse distant weighting, artificial neural

Citation: Salnikov AS (2014) BEHAVIORAL MODEL BUILDING FOR MICROWAVE INTEGRATED CIRCUIT PASSIVE ELEMENTS. ISJ Theoretical \& Applied Science 9 (17): 141-144. doi: http://dx.doi.org/10.15863/TAS.2014.09.17.24
\end{abstract}

\section{УДК 621.382}

\section{ПОСТРОЕНИЕ ПОВЕДЕНЧЕСКИХ МОДЕЛЕЙ ПАССИВНЫХ ЭЛЕМЕНТОВ СВЧ МОНОЛИТНЫХ ИНТЕГРАЛЬНЫХ СХЕМ}

Аннотация: В данной работе приводятся результаты построения параметрических электрических моделей элементов СВЧ монолитных интегральных схем. Для построения модели используются два метода - обратного средневзвешенного расстояния $и$ искусственной нейронной сети. Приводятся результаты построения моделей пассивных элементов: тонкопленочного резистора, МДМ конденсатора, квадратной спиральной катушки индуктивности. Проводится сравнение моделей по точности, быстродействию и сложности построения.

Ключевые слова: элементы СВЧ МИС, поведенческая модель, метод обратного средневзвешенного расстояния, искусственная нейронная сеть

\section{Введение}

Задача построения моделей активных и пассивных элементов (таких, как транзисторы, резисторы, конденсаторы, катушки индуктивности и т.д.) занимает важное место в автоматизированном проектировании СВЧ монолитных интегральных схем (МИС). Важными характеристиками моделей являются точность, быстродействие, а также сложность их построения.

Выделяют несколько видов моделей элементов МИС. Физическое моделирование, учитывающее физические явления и процессы в элементе, требует значительных затрат времени и вычислительных ресурсов. Модели в виде эквивалентных схем (ЭС) являются гораздо более быстродействующими, экономичными и легко встраиваются в современные САПР. Однако способы их построения [1-3] зависят от типа моделируемого элемента МИС и могут быть весьма трудоемкими. Поведенческие модели представляют элемент в виде «черного ящика» без учета его физической природы. Они также быстродействующие, но процедуры их построения не зависят от типа элемента и могут быть формализованы. Поведенческие модели пассивных элементов могут быть построены на основе как 
непосредственных измерений, так и электромагнитного (ЭМ) моделирования характеристик элемента.

В данной работе рассматривается построение поведенческих моделей пассивных элементов СВЧ МИС: тонкопленочного резистора, МДМ конденсатора, квадратной спиральной катушки индуктивности. Для построения моделей используются исходные данные, полученные при помощи ЭМ моделирования на сетке конструктивных состояний в частотном диапазоне 1-40 ГГц.

\section{Получение данных для построения модели с помощью ЭМ моделирования}

В работе [4] предложена новая методика получения данных для построения параметрических моделей. Её основная идея заключается в том, что на основе экстракции простой ЭС небольшого числа изготовленных и измеренных элементов определяются электрофизические параметры, необходимые для ЭМ моделирования. Затем проводится ЭМ моделирование на более частой сетке элементов, что позволяет получить большее количество данных для построения параметрической модели без увеличения числа изготовленных на пластине элементов.

В статье [4] описано применение данной методики для тонкопленочного резистора. Определено удельное сопротивление резистивного слоя и контактное сопротивление и проведено моделирование на сетке конструктивных состояний ширины $w=15,30,45,60$ мкм и длины $l=20,40,60,80,100$ мкм. При применении данной методики для конденсатора определялась относительная диэлектрическая проницаемость, и проводилось моделирование на сетке конструктивных состояний ширина $w=30,50,70,100$ мкм и длина $l=30,50,70,100$ мкм. В статье [5] данная методика была применена для квадратной спиральной катушки индуктивности, определены удельные сопротивление двух слоёв металлизации, используемых для изготовления катушки индуктивности. После чего проведено моделирование катушки на сетке конструктивных состояний ширина $W=10,15$, 20 мкм, зазор между витками $S=5,10$ мкм, количество оборотов спирали $T=1,5,2,5,3,5$.

\section{Построение поведенческих моделей}

Для целей построения поведенческих моделей элементов МИС были использованы два метода многомерной аппроксимации. Аппроксимация с помощью искусственных нейронных сетей (ИНС) [6,7] общеизвестна и широко распространена в разных областях науки и техники. Имеется большое число работ, в которых ИНС использовались при моделировании разных радиоэлектронных элементов и устройств. Метод обратного средневзвешенного расстояния (ОСР) [8,9] - это детерминированный алгоритм интерполяции функций $\mathrm{c}$ неравномерной сеткой данных, легко обобщаемый на произвольную размерность переменных. Значение аппроксимирующей функции в точке $x$ складывается из значений исходной функции в узловых точках $\mathrm{c}$ весовыми коэффициентами, зависящими от расстояния между $x$ и соответствующим узлом. В работе [10] впервые было показано, что метод ОСР может успешно применятся для построения линейной поведенческой модели СВЧ транзистора с достаточной для практики точностью.

Построение поведенческой модели осуществляется путем аппроксимации известных параметров рассеяния элемента СВЧ МИС для различных значений частоты и конструктивных параметров с помощью соответствующего метода (ИНС или ОСР). Действительная и мнимая части каждого элемента матрицы рассеяния аппроксимировались отдельными функциями (всего 8 функций). В качестве входных параметров модели выступает частота и конструктивные параметры элемента (например, длина и ширина резистора).

Для построения модели используется набор файлов, содержащих параметры рассеяния при каждом значении входных параметров модели - обучающая выборка. Для оценки точности модели используются файлы с параметрами рассеяния при других 
значениях входных параметров - контрольная выборка. Результаты оценки точности и быстро приводятся в сводных таблицах, содержащих данные по максимальному и среднему значению среднеквадратичной ошибки построенной модели для файлов контрольной выборки, количеству файлов обучающей выборки с точностью выше определённого значения, времени построения и расчёта характеристик модели.

\section{Результаты построения поведенческих моделей}

Тонкопленочный резистор. При построении поведенческой модели использовалась обучающая выборка из 16 файлов и тестовая выборка из 4 файлов с результатами электрофизического моделирования параметров рассеяния элемента в разных конструктивных состояниях. Входными переменными модели являлись рабочая частота, длина и ширина резистора. Результаты оценки точности и быстродействия поведенческих моделей тонкопленочного резистора приведены в табл. 1.

Таблица 1

Оценка точности и быстродействия поведенческих моделей тонкопленочного резистора

\begin{tabular}{|l|l|l|l|l|l|l|l|}
\hline Метод & $\begin{array}{l}\text { Максимальная } \\
\text { СКО }\end{array}$ & $\begin{array}{l}\text { Средняя } \\
\text { СКО }\end{array}$ & & \multicolumn{2}{l}{$\begin{array}{l}\text { Число файлов с СКО не } \\
\text { более }\end{array}$} & $\begin{array}{l}\text { Время } \\
\text { построения } \\
\text { модели }\end{array}$ & $\begin{array}{l}\text { Время } \\
\text { расчета } \\
\text { одного } \\
\text { файла }\end{array}$ \\
\hline ИНС & $2,27 \%$ & & $5 \%$ & $1 \%$ & & $2,2 \mathrm{мc}$ \\
\hline ОСР & $4,49 \%$ & $1,33 \%$ & 4 & 4 & 2 & 12,789 с. & $2,2 \%$ \\
\hline
\end{tabular}

МДМ конденсатор. При построении поведенческой модели использовалась обучающая выборка из 24 файлов и тестовая выборка из 4 файлов с результатами электрофизического моделирования параметров рассеяния элемента в разных конструктивных состояниях. Входными переменными модели являлись рабочая частота, длина и ширина верхней обкладки конденсатора. Результаты оценки точности и быстродействия поведенческих моделей МДМ конденсатора приведены в табл. 2.

Таблица 2

Оценка точности и быстродействия поведенческих моделей МДМ конденсатора

\begin{tabular}{|c|c|c|c|c|c|c|c|}
\hline \multirow[t]{2}{*}{ Метод } & \multirow[t]{2}{*}{$\begin{array}{l}\text { Максимальная } \\
\text { СКО }\end{array}$} & \multirow[t]{2}{*}{$\begin{array}{l}\text { Средняя } \\
\text { СКО }\end{array}$} & \multicolumn{3}{|c|}{$\begin{array}{l}\text { Число файлов с СКО не } \\
\text { более }\end{array}$} & \multirow{2}{*}{$\begin{array}{l}\text { Время } \\
\text { построения } \\
\text { модели }\end{array}$} & \multirow{2}{*}{$\begin{array}{l}\text { Время } \\
\text { расчета } \\
\text { одного } \\
\text { файла }\end{array}$} \\
\hline & & & $10 \%$ & $5 \%$ & $1 \%$ & & \\
\hline ИНС & $81,2 \%$ & $25,8 \%$ & 2 & 2 & 0 & 1 мин. 39,6 с. & 0,9 мс. \\
\hline $\mathrm{OCP}$ & $85,1 \%$ & $26,9 \%$ & 2 & 2 & 0 & $0,438 \mathrm{c}$. & 2,3 мc. \\
\hline
\end{tabular}

Можно заключить, что при построении поведенческой модели МДМ конденсатора данные методы дают неудовлетворительные результаты. Причина проблемной работы алгоритмов на данный момент автору не известна.

Квадратная спиральная катушка индуктивности. При построении поведенческой модели использовалась обучающая выборка из 14 файлов и тестовая выборка из 4 файлов с результатами электрофизического моделирования параметров рассеяния элемента в разных конструктивных состояниях. Входными переменными модели являлись рабочая частота, ширина спирали, зазор между витками и количество витков спирали. Результаты оценки точности и быстродействия поведенческих моделей квадратной спиральной катушки индуктивности приведены в табл. 3.

Таблица 3

Оценка точности и быстродействия поведенческих моделей квадратной спиральной катушки индуктивности

\begin{tabular}{|l|l|l|l|l|l|}
\hline Метод & $\begin{array}{l}\text { Максимальная } \\
\text { СКО }\end{array}$ & $\begin{array}{l}\text { Средняя } \\
\text { СКО }\end{array}$ & $\begin{array}{l}\text { Число файлов с СКО не } \\
\text { более }\end{array}$ & $\begin{array}{l}\text { Время } \\
\text { построения }\end{array}$ & $\begin{array}{l}\text { Время } \\
\text { расчета }\end{array}$ \\
\hline
\end{tabular}




\begin{tabular}{|l|l|l|l|l|l|l|l|}
\hline & & & $10 \%$ & $5 \%$ & $1 \%$ & модели & $\begin{array}{l}\text { одного } \\
\text { файла }\end{array}$ \\
\hline ИНС & 26,1 & 14,0 & 2 & 0 & 0 & 1 мин. 43,8 с. & 1,95 мс. \\
\hline ОСР & 30,8 & 14,3 & 2 & 0 & 0 & 2,44 с. & 14,4 мс. \\
\hline
\end{tabular}

Поведенческая модель на основе ОСР была интегрирована в САПР, где проведено сравнительное исследование быстродействия. Исследование показало, что среднее время расчёта поведенческих моделей составляет 0,02 секунды. Среднее время расчёта широко распространенных моделей в виде эквивалентных схем в той же САПР составляет 0,01 с. Таким образом, поведенческая модель обладает меньшей скоростью расчёта, чем модель в виде ЭС, что является их недостатком. Однако процедура построения поведенческой модели является универсальной для всех элементов, более простой и легко автоматизируемой. Поэтому рекомендуется использовать поведенческие модели для более сложных элементов, например, транзисторов.

\section{Заключение}

Показана работоспособность метода обратного средневзвешенного расстояния для построения пассивных и элементов: тонкопленочного резистора, МДМ конденсатора спиральной катушки индуктивности. Точность построенных моделей сопоставима с точностью моделей в виде искусственных нейронных сетей. Проведенные исследования показывают, что метод ОСР может успешно использоваться для построения поведенческих моделей элементов СВЧ МИС.

\section{References:}

1. Yan N, Yang C, Gao J (2013) An approach for determining equivalent circuit model of onchip inductors. Microwave and optical technology letters. Vol. 55, No.10. pp. 2363-2370.

2. Shih YC, Pao CK, Itoh T (1992) A broadband parameter extraction technique for the equivalent circuit of planar inductors. IEEE Microwave Symposium Digest, Vol. 3, pp. 13451348 .

3. Goryainov AE and other (2012) Programma dlya ekstraktsii parametrov ekvivalentnykh skhem passivnykh komponentov SVCh monolitnykh integral'nykh skhem. Mat. 22-oy Mezhdunarodnoy Krymskoy konferentsii «SVCh-tekhnika i telekommunikatsionnye tekhnologii». Sevastopol, Veber, T.1, pp. 129-130.

4. Salnikov AS, Dobush IM, Goryainov AE, Babak LI (2014) Postroenie parametricheskoy modeli monolitnogo tonkoplenochnogo rezistora na osnove SVCh izmereniy i elektromagnitnogo modelirovaniya. Doklady TUSUR. v pechati.

5. Salnikov AS, Babak LI (2014) Opredelenie fizicheskikh parametrov monolitnoy katushki induktivnosti na osnove rezul'tatov SVCh izmereniy. Mat-ly konf. Aktual'nye problemy elektronnogo priborostroeniya APEP-2014. T.4. v pechati.

6. Aksenov SV, Novosel'tsev VB (2006) Organizatsiya i ispol'zovanie neyronnykh setey. Metody i tekhnologii. Tomsk: Izd-vo NTL, pp.126.

7. Kallan R (2001) Osnovnye kontseptsii neyronnykh setey. Moscow, Vil'yamc, pp.287.

8. Shepard D (1968) A two-dimensional interpolation function for irregularly-spaced data. Proceedings of the 23rd ACM national conference, pp.517-524.

9. Renka RJ (1998) Multivariate interpolation of large sets of scattered data. ACM Transactions on Mathematical Software. Vol. 14, No.2, pp.139-148.

10. Salnikov AS (2014) Issledovanie povedencheskikh modeley elementov SVCh MIS. Mat-ly konf. 24-ya Mezhdunarodnaya Krymskaya konferentsiya «SVCh-tekhnika i telekommunikatsionnye tekhnologii» (KryMiKo). T.1, pp.119-120. 